\title{
AN EMBEDDING RELATION FOR BOUNDED MEAN OSCILLATION ON RECTANGLES.
}

\author{
BENOÎT F. SEHBA
}

\begin{abstract}
In the two-parameter setting, we say a function belongs to the mean little BMO, if its mean over any interval and with respect to any of the two variables has uniformly bounded mean oscillation. This space has been recently introduced by S. Pott and the author in relation with the multiplier algebra of the product BMO of Chang-Fefferman. We prove that the Cotlar-Sadosky space of functions of bounded mean oscillation bmo $\left(\mathbb{T}^{N}\right)$ is a strict subspace of the mean little BMO.
\end{abstract}

\section{IntRoduction AND RESUlts}

1.1. Introduction. In the two-parameter case, the mean little BMO space consists of those functions such that their mean over any interval with respect to any of the two variables is uniformly in $\operatorname{BMO}(\mathbb{T})$. This space was introduced recently in the literature by S. Pott and the author in their way to the characterization of the multiplier algebra of the product BMO of Chang-Fefferman $([1,6,6])$. Its definition is very close in spirit to the one of the little BMO of Cotlar and Sadosky ([2]) and this is somehow misleading. It is pretty clear that the little BMO embeds continuously into the mean little BMO and it was natural to ask if both spaces are the same. To find out, we use an indirect method; we characterize the multiplier algebra of the Cotlar-Sadosky space and the set of multipliers from the little $B M O$ to the mean little $B M O$.

1.2. Definitions and results. Given two Banach spaces of functions $X$ and $Y$, the space of pointwise multipliers from $X$ to $Y$ is defined as follows

$$
\mathcal{M}(X, Y)=\{\phi: \phi f \in Y \text { for all } f \in X\}
$$

When $X=Y$, we simply write $\mathcal{M}(X, X)=\mathcal{M}(X)$.

The so-called small BMO space on $\mathbb{T}^{N}$, introduced by Cotlar and Sadosky and denoted bmo $\left(\mathbb{T}^{N}\right)$ consists of functions $b \in L^{2}\left(\mathbb{T}^{N}\right)$ such that the

2010 Mathematics Subject Classification. Primary 42B15, 32A37; Secondary 42B35.

Key words and phrases. Bounded mean oscillation, logarithmic mean oscillation, product domains. 
quantity

$$
\|b\|_{*, N}:=\sup _{R \subset \mathbb{T}^{N}, \text { rectangle }} \frac{1}{|R|} \int_{R}\left|b\left(t_{1}, \cdots, t_{N}\right)-m_{R} b\right| d t_{1} \cdots d t_{N}
$$

is finite, $m_{R} b=\frac{1}{|R|} \int_{R} b\left(t_{1}, \cdots, t_{N}\right) d t_{1} \cdots d t_{N}$. Seen as a quotient space with the set of constants, bmo $\left(\mathbb{T}^{N}\right)$ is a Banach space with norm $\|b\|_{\mathrm{bmo}\left(\mathbb{T}^{N}\right)}:=$ $\|b\|_{*, N}$.

Note that in the above definition, since $R$ is a rectangle in $\mathbb{T}^{N}, m_{R} f$ is a constant. We will sometimes consider the case where $R$ is a rectangle in $\mathbb{T}^{M}$ with $M$ an integer, $0<M<N$, in which case $m_{R} f$ is a function of $N-M$ variables.

Another notion of function of bounded mean oscillation was introduced in [6] in the two-parameter setting. This notion is inspired from the one of M. Cotlar and C. Sadosky ([2]). One of its higher-parameter versions is defined as follows.

Definition 1.1. A function $b \in L^{2}\left(\mathbb{T}^{N}\right)$ belongs to $\operatorname{bmo}_{m}\left(\mathbb{T}^{N}\right)$ if there is a constant $C>0$ such that for any integers $0<N_{1}, N_{2}<N, N_{1}+N_{2}=N$ and any rectangle $R \subset \mathbb{T}^{N_{1}}$,

$$
\left\|m_{R} b\right\|_{*, N_{2}} \leq C
$$

The space $\mathrm{bmo}_{m}\left(\mathbb{T}^{N}\right)$ seen as a quotient space by the set of constants is a Banach space under the norm

$$
\|b\|_{\mathrm{bmo}_{m}\left(\mathbb{T}^{N}\right)}:=C^{*}
$$

where $C^{*}$ stands for the smallest constant in the above definition.

It is clear from the definitions above that bmo $\left(\mathbb{T}^{N}\right)$ embeds continuously into $\mathrm{bmo}_{m}\left(\mathbb{T}^{N}\right)$. We will be calling $\mathrm{bmo}_{m}$ the mean little BMO. Our main result is the following.

Theorem 1.2. $\operatorname{bmo}\left(\mathbb{T}^{N}\right)$ is strictly continuously embedded into bmo $\mathrm{bm}^{N}\left(\mathbb{T}^{N}\right)$.

To prove the above theorem, we first prove the following.

Theorem 1.3. The only pointwise multipliers of $\operatorname{bmo}\left(\mathbb{T}^{N}\right)$ are the constants.

We say a function $b \in L^{2}\left(\mathbb{T}^{N}\right)$ has bounded logarithmic mean oscillation on rectangles, i.e $b \in \operatorname{lmo}\left(\mathbb{T}^{N}\right)$ if

$$
\begin{aligned}
\|b\|_{*, \log , N} & :=\sup _{\substack{R=I_{1} \times \cdots \times I_{N} \subset \mathbb{T}^{N}\\
}} \frac{\sum_{j=1}^{N} \log \frac{4}{\left|I_{j}\right|}}{|R|} \int_{R}\left|b(t)-m_{R} b\right| d t \\
& <.
\end{aligned}
$$

Let us introduce also the mean little LMO space in product domains. 
Definition 1.4. A function $b \in L^{2}\left(\mathbb{T}^{N}\right)$ belongs to $\operatorname{lmo}_{m}\left(\mathbb{T}^{N}\right)$ if there is a constant $C>0$ such that for any decomposition $0<N_{1}, N_{2}<N, N_{1}+N_{2}=$ $N$, and any rectangle $R \subset \mathbb{T}^{N_{1}}$,

$$
\left\|m_{R} b\right\|_{*, \log , N_{2}} \leq C .
$$

If $C^{*}$ stands for the smallest constant in the Definition 1.4, then seen as a quotient space by the set of constants, $\operatorname{lmo}_{m}\left(\mathbb{T}^{N}\right)$ is a Banach space with the following norm

$$
\|b\|_{\operatorname{lmo}_{m}\left(\mathbb{T}^{N}\right)}:=C^{*}
$$

In terms of multipliers, to get close to the one parameter situation, we need to start from $\operatorname{bmo}\left(\mathbb{T}^{N}\right)$ and take $\operatorname{bmo}_{m}\left(\mathbb{T}^{N}\right)$ as the target space.

Theorem 1.5. Let $\phi \in L^{2}\left(\mathbb{T}^{N}\right)$. Then the following assertions are equivalent.

(i) $\phi$ is a multiplier from $\mathrm{bmo}\left(\mathbb{T}^{N}\right)$ to $\mathrm{bmo}_{m}\left(\mathbb{T}^{N}\right)$.

(ii) $\phi \in \operatorname{lmo}_{m}\left(\mathbb{T}^{N}\right) \cap L^{\infty}\left(\mathbb{T}^{N}\right)$.

Moreover,

$$
\left\|M_{\phi}\right\|_{\mathrm{bmo}\left(\mathbb{T}^{N}\right) \rightarrow \mathrm{bmo}_{m}\left(\mathbb{T}^{N}\right)} \simeq\|\phi\|_{L^{\infty}\left(\mathbb{T}^{N}\right)}+\|\phi\|_{\operatorname{lmo}_{m}\left(\mathbb{T}^{N}\right)}
$$

where $\left\|M_{\phi}\right\|_{\mathrm{bmo}\left(\mathbb{T}^{N}\right) \rightarrow \mathrm{bmo}_{m}\left(\mathbb{T}^{N}\right)}$ is the norm of the multiplication operator from $\operatorname{bmo}\left(\mathbb{T}^{N}\right)$ to $\mathrm{bmo}_{m}\left(\mathbb{T}^{N}\right)$.

Theorem 1.3 and Theorem 1.5 clearly establish Theorem 1.2 since $\operatorname{lmo}_{m}\left(\mathbb{T}^{N}\right) \cap L^{\infty}\left(\mathbb{T}^{N}\right)$ contains more than constants. The proofs are given in the next section. The last section of this note also states that the only multiplier from a Banach space of functions (strictly) containing bmo $\left(\mathbb{T}^{N}\right)$ to $\operatorname{bmo}\left(\mathbb{T}^{N}\right)$ is the constant zero.

As we are dealing only with little spaces of functions of bounded mean oscillation, we essentially make use of the one parameter techniques. This is not longer possible when considering the multipliers of the product BMO of Chang-Fefferman for which one needs more demanding techniques $([4,5,6])$.

\section{Comparison via multiplier algebras}

2.1. Proof of Theorem 1.3. The space bmo $\left(\mathbb{T}^{N}\right)$ has the following equivalent definitions $([2,3])$ that we need here.

Proposition 2.1. The following assertions are equivalent.

(1) $b \in \operatorname{bmo}\left(\mathbb{T}^{N}\right)$.

(2) $b \in L^{2}\left(\mathbb{T}^{N}\right)$ and there exists a constant $C>0$ such that for any decomposition $N_{1}+N_{2}=N, 0<N_{1}, N_{2}<N$, 
(i) $\|b(\cdot, t)\|_{*, N_{1}} \leq C$, for all $t \in \mathbb{T}^{N_{2}}$.

(ii) $\|b(s, \cdot)\|_{*, N_{2}} \leq C$ for all $s \in \mathbb{T}^{N_{1}}$.

Proof. The proof was given in the two-parameter case in [2]. It is essentially the same proof in the multi-parameter setting. We follow the simplified two-parameter proof from [8].

We first suppose that $b \in \operatorname{bmo}\left(\mathbb{T}^{N}\right)$ that is we have that for any $S \subset \mathbb{T}^{N_{1}}$, $K \subset \mathbb{T}^{N_{2}}, N_{1}+N_{2}=N$,

$$
\frac{1}{|S|} \frac{1}{|K|} \int_{S} \int_{K}\left|b(s, t)-m_{S \times K} b\right| d s d t \leq\|b\|_{*, N} .
$$

If $S=S_{1} \times \cdots \times S_{N_{1}}$, then letting $\left|S_{1}\right| \rightarrow 0$ we get that

$$
\frac{1}{\left|S^{\prime}\right||K|} \int_{K} \int_{S^{\prime}}\left|b(s, t)-m_{K \times S^{\prime}} b\right| d s d t \leq\|b\|_{*, N},
$$

for any $S^{\prime}=S_{2} \times \cdots \times S_{N_{1}} \subset \mathbb{T}^{N_{1}-1}$.

Repeating this process for $S_{2}, \cdots, S_{N_{1}}$, we obtain that

$$
\frac{1}{|K|} \int_{K}\left|b(s, t)-m_{K} b\right| d t \leq\|b\|_{*, N}
$$

and consequently that

$$
\sup _{s \in \mathbb{T}^{N_{1}}}\|b(s, \cdot)\|_{*, N_{2}} \leq\|b\|_{*, N} .
$$

The same reasoning leads to

$$
\sup _{t \in \mathbb{T}^{N_{2}}}\|b(\cdot, t)\|_{*, N_{1}} \leq\|b\|_{*, N}
$$

For the converse, we write $b(s, t)-m_{S \times K} b$ as follows

$$
b(s, t)-m_{S \times K} b=\left(b(s, t)-m_{K} b(s)\right)+\left(m_{K} b(s)-m_{S \times K} b\right) .
$$

Hence

$$
\left|b(s, t)-m_{S \times K} b\right| \leq\left|b(s, t)-m_{K} b(s)\right|+\left|m_{K} b(s)-m_{S \times K} b\right| .
$$

Integrating both sides of (2.1) over $S \times K$ and with respect to the measure $\frac{d s d t}{|S||K|}$, we obtain

$$
\begin{aligned}
L:= & \frac{1}{|S||K|} \int_{S} \int_{K}\left|b(s, t)-m_{S \times K} b\right| d s d t \\
\leq & \frac{1}{|S||K|} \int_{S} \int_{K}\left|b(s, t)-m_{K} b(s)\right| d s d t \\
& +\frac{1}{|S||K|} \int_{S} \int_{K}\left|m_{K} b(s)-m_{S \times K} b\right| d s d t \\
= & L_{1}+L_{2} .
\end{aligned}
$$


Clearly,

$$
\begin{aligned}
L_{1} & :=\frac{1}{|S||K|} \int_{S} \int_{K}\left|b(s, t)-m_{K} b(s)\right| d s d t \\
& \leq \frac{1}{|S|} \int_{S}\left(\frac{1}{|K|} \int_{K}\left|b(s, t)-m_{K} b(s)\right| d t\right) d s \\
& \leq \frac{1}{|S|} \int_{S}\|b(s, \cdot)\|_{*, N_{2}} d s \leq C .
\end{aligned}
$$

On the other hand,

$$
\begin{aligned}
L_{2} & :=\frac{1}{|S||K|} \int_{S} \int_{K}\left|m_{K} b(s)-m_{S \times K} b\right| d s d t \\
& =\frac{1}{|S|} \int_{S}\left|m_{K} b(s)-m_{S \times K} b\right| d s \\
& =\frac{1}{|S|} \int_{S}\left|\frac{1}{|K|} \int_{K}\left(b(s, t)-m_{S} b(t)\right) d t\right| d s \\
& \leq \frac{1}{|S||K|} \int_{S} \int_{K}\left|b(s, t)-m_{S} b(t)\right| d s d t \\
& =\frac{1}{|K|} \int_{K}\left(\frac{1}{|S|} \int_{S}\left|b(s, t)-m_{S} b(t)\right| d s\right) d t \\
& \leq \frac{1}{|K|} \int_{K}\|b(\cdot, t)\|_{*, N_{1}} d t \leq C .
\end{aligned}
$$

Thus for any $S \in \mathbb{T}^{N_{1}}$ and $K \in \mathbb{T}^{N_{2}}$,

$$
\frac{1}{|S||K|} \int_{S} \int_{K}\left|b(s, t)-m_{S \times K} b\right| d s d t \leq 2 C .
$$

Hence $\|b\|_{*, N}<\infty$. The proof is complete.

Note that if $C^{*}$ is the smallest constant in the equivalent definition above, then $C^{*}$ is comparable to \|\|$_{\mathrm{bmo}\left(\mathbb{T}^{N}\right)}$.

We make the following observation that can be proved exactly as in the one parameter case.

Lemma 2.2. Let $b \in L^{2}\left(\mathbb{T}^{N}\right)$. Then

$$
\|b\|_{\mathrm{bmo}\left(\mathbb{T}^{N}\right)} \simeq\|b\|_{N}^{*}:=\sup _{R \subset \mathbb{T}^{N}} \inf _{\lambda \in \mathbb{C}} \frac{1}{|R|} \int_{R}|b(t)-\lambda| d t .
$$

Let us also observe the following.

Lemma 2.3. The following assertions hold.

(i) Given an interval $I$ in $\mathbb{T}$, there is a function in $\mathrm{BMO}(\mathbb{T})$, denoted $\log _{I}$ such that

- the restriction of $\log _{I}$ to $I$ is $\log \frac{4}{|I|}$. 
- $\left\|\log _{I}\right\|_{\mathrm{BMO}(\mathbb{T})} \leq C$ where $C$ is a constant that does not depend on $I$.

(ii) For any $f_{1}, \cdots, f_{N} \in \mathrm{BMO}(\mathbb{T})$, the function

$b\left(t_{1}, \cdots, t_{N}\right)=\sum_{j=1}^{N} f_{j}\left(t_{j}\right)$ belongs to bmo $\left(\mathbb{T}^{N}\right)$. Moreover,

$$
\|b\|_{\mathrm{bmo}\left(\mathbb{T}^{N}\right)} \leq \sum_{j=1}^{N}\left\|f_{j}\right\|_{\mathrm{BMO}(\mathbb{T})} .
$$

(iii) There is a constant $C>0$ such that for any $b \in \mathrm{bmo}_{m}\left(\mathbb{T}^{N}\right)$ and any rectangle $R=I_{1} \times \cdots \times I_{N} \subset \mathbb{T}^{N}$,

$$
\left|m_{R} b\right| \leq C\left(\log \frac{4}{\left|I_{1}\right|}+\cdots+\log \frac{4}{\left|I_{N}\right|}\right)\|b\|_{\mathrm{bmo}_{m}\left(\mathbb{T}^{N}\right)},
$$

and this is sharp.

Proof. Assertion (ii) follows directly from the definition of $\mathrm{bmo}\left(\mathbb{T}^{N}\right)$.

(i) is surely well known, we give a proof here for completeness: let $J$ be a fixed interval in $\mathbb{T}$. Let $J_{0}=J$ and $J_{k}$ be the intervals in $\mathbb{T}$ with the same center as $J$ and such that $\left|J_{k}\right|=2^{k}|J|$, here $k=1,2, \cdots, N-1$ and $N$ is the smallest integer such that $2^{N}|J| \geq 1$. We define $J_{N}=\mathbb{T}$. Thus,

$$
N \leq \log _{2} \frac{4}{|J|} \leq N+2 .
$$

Next, we define $U_{0}=J_{0}=J, U_{k}=J_{k} \backslash J_{k-1}$, for $k=1, \cdots, N$. Now consider the function $\log _{J}$ defined on $\mathbb{T}$ by

$$
\log _{J}(t)=\sum_{k=0}^{N}(N+2-k) \chi_{U_{k}}(t), \quad t \in \mathbb{T} .
$$

Clearly,

$$
\log _{J}(t)=N+2 \simeq \log _{2} \frac{4}{|J|} \text { for all } t \in J .
$$

Lemma 2.4. For each interval $J \subset \mathbb{T}$, the function $\log _{J}$ defined by (2.3) belongs to $B M O(\mathbb{T})$.

Proof. We start by estimating the $L^{2}$-norm of $\log _{J}$. We have

$$
\begin{aligned}
\left\|\log _{J}\right\|_{2}^{2} & =\sum_{k=0}^{N}(N+2-k)^{2}\left|U_{k}\right|=\sum_{k=2}^{N+2} k^{2}\left|J_{N+2-k}\right| \\
& \leq \sum_{k=1}^{N+2} k^{2} 2^{N+2-k}|J| \leq \sum_{k=1}^{N+2} k^{2} 2^{N+2-k} 2^{1-N} \\
& =8 \sum_{k=1}^{N+2} k^{2} 2^{-k} .
\end{aligned}
$$


It is clear that the last sum in the above equalities is finite and so $\log _{J} \in$ $L^{2}(\mathbb{T})$.

For any dyadic interval $I \subset \mathbb{T}$, let $m \in\{0, \cdots, N+1\}$ be minimal such that $I \cap U_{m} \neq \emptyset$, and $l \in\{0, \cdots, N+1\}$ be maximal such that $I \cap U_{m+l} \neq \emptyset$. Let us estimate the length of $I \cap U_{j}$ for any $m \leq j \leq m+l$.

If $l=1$ then $I \cap U_{m}=I$ and there is nothing to say. If $l=2$ then $\left|I \cap U_{m}\right| \leq|I|$ and $\left|I \cap U_{m+1}\right| \leq|I|$.

Next, we consider the case $l \geq 2$. We remark that in this case, half of $U_{m+l-1}$ is contained in $I$. Consequently, for any $m \leq j<m+l$, we have $\left|I \cap U_{j}\right| \leq 2 \frac{1}{2^{m+l-j-1}}|I|$. Finally, we have

$$
\left|I \cap U_{m+l}\right| \leq 2\left|I \cap U_{m+l-1}\right| \leq 2|I| .
$$

Hence,

$$
\begin{aligned}
L & :=\frac{1}{|I|} \int_{I}\left|\log _{J}-(N+2-m-l)\right| d t \\
& =\frac{1}{|I|} \int_{I}\left|\sum_{k=m}^{m+l}(m+l-k) \chi_{U_{k}}\right| d t \\
& \leq \frac{1}{|I|} \sum_{k=m}^{m+l}(m+l-k)\left|I \cap U_{k}\right| \\
& \leq 4 \frac{1}{|I|} \sum_{k=m}^{m+l}(m+l-k) 2^{-m-l+k}|I| \\
& =4 \sum_{k=0}^{k=l} \frac{k}{2^{k}} \leq 6 .
\end{aligned}
$$

Thus, for each interval $J \subset \mathbb{T}$, the function $\log _{J}$ given by (2.3) belongs to $B M O(\mathbb{T})$ and there exists a positive constant $C$ independent of $J$ such that $\left\|\log _{J}\right\|_{B M O(\mathbb{T})} \leq C$.

To prove (iii), we observe that by definition, given $b \in \mathrm{bmo}_{m}\left(\mathbb{T}^{N}\right)$, for any rectangle $S \subset \mathbb{T}^{K}, 0<K<N,\left\|m_{S} b\right\|_{*, N-K}$ is uniformly bounded. It follows from the one parameter estimate of the mean of a function of bounded mean oscillation and the definition of $\mathrm{bmo}_{m}\left(\mathbb{T}^{N}\right)$ that for any rectangle $Q \subset \mathbb{T}^{N-1}$,

$$
\begin{aligned}
\left|m_{I}\left(m_{Q} b\right)\right| & \lesssim\left(\log \frac{4}{|I|}\right)\left\|m_{Q} b\right\|_{*, 1} \\
& \lesssim\left(\log \frac{4}{|I|}\right)\|b\|_{\mathrm{bmo}_{m}\left(\mathbb{T}^{N}\right)} .
\end{aligned}
$$


In particular, for any rectangle $R=I_{1} \times \cdots \times I_{N} \subset \mathbb{T}^{N}$, we have

$$
\left|m_{R} b\right| \leq C\left(\sum_{j=1}^{N} \log \frac{4}{\left|I_{j}\right|}\right)\|b\|_{\mathrm{bmo}_{m}\left(\mathbb{T}^{N}\right)}
$$

The sharpness follows by applying the last inequality to the function $\log _{R}\left(t_{1}, \cdots, t_{N}\right)=\sum_{j=1}^{N} \log _{I_{j}}\left(t_{j}\right), R=I_{1} \times \cdots \times I_{N}$, and using (ii).

The proof is complete.

Lemma 2.4 and its proof complete the proof of Lemma 2.3,

We now reformulate and prove Theorem 1.3 ,

Theorem 2.5. Let $\phi \in L^{2}\left(\mathbb{T}^{N}\right)$. Then the following assertions are equivalent.

(a) $\phi$ is multiplier of bmo $\left(\mathbb{T}^{N}\right)$.

(b) $\phi$ is a constant.

Proof. Clearly, (b) $\Rightarrow$ (a). We prove that (a) $\Rightarrow(\mathrm{b})$.

Assume that $\phi \in L^{2}\left(\mathbb{T}^{N}\right)$ is a multiplier of $\operatorname{bmo}\left(\mathbb{T}^{N}\right)$. Then for any $f \in$ $\operatorname{bmo}\left(\mathbb{T}^{N}\right)$, and any integer $0<N_{1}<N, N_{2}=N-N_{1},\|(\phi f)(., t)\|_{*, N_{1}}$ is uniformly bounded for all $t \in \mathbb{T}^{N_{2}}$ fixed and $\|(\phi f)(., t)\|_{*, N_{1}} \leq\|\phi f\|_{\mathrm{bmo}\left(\mathbb{T}^{N}\right)}$. Let us take as $f$ the function $f(s, t)=\log _{R}(s, t)=\sum_{k=1}^{N_{1}} \log _{S_{k}}(s)+\sum_{j=1}^{N_{2}} \log _{Q_{j}}(t)$, $R=S \times Q \subset \mathbb{T}^{N_{1}} \times \mathbb{T}^{N_{2}}, S=S_{1} \times \cdots \times S_{N_{1}}, Q=Q_{1} \times \cdots \times Q_{N_{2}}$, $S_{k} \subset \mathbb{T}, Q_{j} \subset \mathbb{T}$. Then it follows that

$$
\frac{1}{|S|} \int_{S}\left|\phi(s, t) f(s, t)-m_{S}(\phi f)\right| d s \leq\|\phi f\|_{\mathrm{bmo}\left(\mathbb{T}^{N}\right)}, \text { for all } S \subset \mathbb{T}^{N_{1}} \text {. }
$$

But from assertion (i) of Lemma 2.3 we have that for any $t \in Q \subset \mathbb{T}^{N_{2}}$ fixed,

$$
\begin{aligned}
L & :=\frac{\sum_{j=1}^{N_{2}} \log \frac{4}{\left|Q_{j}\right|}+\sum_{k=1}^{N_{1}} \log \frac{4}{|S|}}{|S|} \int_{S}\left|\phi(s, t)-m_{S} \phi\right| d s \\
& \lesssim \frac{1}{|S|} \int_{S}\left|\phi(s, t) \log _{R}(s, t)-m_{S}\left(\phi \log _{R}\right)\right| d s \\
& \leq\left\|\phi \log _{R}\right\|_{\mathrm{bmo}\left(\mathbb{T}^{N}\right)} \\
& \lesssim\left\|M_{\phi}\right\|,
\end{aligned}
$$

where $\left\|M_{\phi}\right\|$ is the norm of the multiplication by $\phi, M_{\phi}(f)=\phi f$.

Hence for any $S \subset \mathbb{T}^{N_{1}}, Q \subset \mathbb{T}^{N_{2}}$ and $t \in Q$,

$$
\left(\sum_{j=1}^{N_{2}} \log \frac{4}{\left|Q_{j}\right|}+\sum_{k=1}^{N_{1}} \log \frac{4}{\left|S_{k}\right|}\right)\left(\frac{1}{|S|} \int_{S}\left|\phi(s, t)-m_{S} \phi\right| d s\right)<\infty .
$$


Letting for example $\left|Q_{1}\right| \rightarrow 0$ in (2.4), we see that necessarily, $\phi(s, t)=\phi(t)$ for any $s \in S \subset \mathbb{T}^{N_{1}}$. As $N_{1}$ runs through $(0, N)$, we obtain that for any $\left(t_{1}, \cdots, t_{N}\right) \in \mathbb{T}^{N}$,

$$
\phi\left(t_{1}, \cdots, t_{N}\right)=\phi\left(t_{j}\right)=\phi\left(t_{j_{1}}, \cdots, t_{j_{k}}\right), \quad j, j_{l} \in\{1, \cdots, N\},
$$

$0<k<N$. The latter gives that $\phi$ is a constant.

We have the following consequence which says that the only bounded functions in $\operatorname{lmo}\left(\mathbb{T}^{N}\right)$ are the constants. This is pretty different from the one parameter case $([7])$.

Corollary 2.6. Assume that $\phi \in L^{\infty}\left(\mathbb{T}^{N}\right)$ and

$$
\|\phi\|_{*, \log , N}:=\sup _{R=I_{1} \times \cdots \times I_{N} \subset \mathbb{T}^{N}} \frac{\sum_{j=1}^{N} \log \frac{4}{\left|I_{j}\right|}}{|R|} \int_{R}\left|\phi(t)-m_{R} \phi\right| d t<\infty .
$$

Then $\phi$ is a constant.

Proof. Following Theorem 1.3 we only need to prove that any bounded function $\phi$ which satisfies (2.5) is a multiplier of $\operatorname{bmo}\left(\mathbb{T}^{N}\right)$. For this we first recall that if $f \in \operatorname{bmo}\left(\mathbb{T}^{N}\right)$, then for any rectangle $R=I_{1} \times \cdots \times I_{N} \subset \mathbb{T}^{N}$, we have the estimate

$$
\left|m_{R} f\right| \lesssim\left(\log \frac{4}{\left|I_{1}\right|}+\cdots+\log \frac{4}{\left|I_{N}\right|}\right)\|f\|_{\mathrm{bmo}\left(\mathbb{T}^{N}\right)} .
$$

Now assume that $\phi \in L^{\infty}\left(\mathbb{T}^{N}\right)$ and satisfies (2.5), and let $f \in \operatorname{bmo}\left(\mathbb{T}^{N}\right)$. Then using the above estimate, we obtain for any $R=I_{1} \times \cdots \times I_{N} \subset \mathbb{T}^{N}$,

$$
\begin{aligned}
& \frac{1}{|R|} \int_{R}\left|(f \phi)(t)-m_{R} \phi m_{R} f\right| d t \\
\leq & \frac{1}{|R|} \int_{R}|\phi(t)|\left|f(t)-m_{R} f\right| d t+ \\
& \frac{1}{|R|} \int_{R}\left|m_{R} f \| \phi(t)-m_{R} \phi\right| d t \\
\leq & \frac{\| \phi \mid L_{L^{\infty}\left(\mathbb{T}^{N}\right)}}{|R|} \int_{R}\left|f(t)-m_{R} f\right| d t+ \\
& \frac{\|f \mid\|_{\mathrm{bmo}\left(\mathbb{T}^{N}\right)}\left(\log \frac{4}{\left|I_{1}\right|}+\cdots+\log \frac{4}{\left|I_{N}\right|}\right)}{|R|} \int_{R}\left|\phi(t)-m_{R} \phi\right| d t \\
\leq & \left(\|\phi\|_{L^{\infty}\left(\mathbb{T}^{N}\right)}+\|\phi\|_{*, \log , N}\right)\|f\|_{\mathrm{bmo}\left(\mathbb{T}^{N}\right)} .
\end{aligned}
$$

It follows from the latter and Lemma 2.2 that if $\phi$ is bounded and satisfies (2.5), then for any $f \in \operatorname{bmo}\left(\mathbb{T}^{N}\right), \phi f$ belongs to $\operatorname{bmo}\left(\mathbb{T}^{N}\right)$. That is $\phi$ is a multiplier of $\operatorname{bmo}\left(\mathbb{T}^{N}\right)$. The proof is complete. 
Remark 2.7. Let us first recall that in the one parameter case, it is a result of D. Stegenga [7] that $L^{\infty}(\mathbb{T}) \cap \operatorname{LMO}(\mathbb{T})$ is the exact range of pointwise multipliers of $\mathrm{BMO}(\mathbb{T})$. Let us define another little LMO space in the twoparameter case $\operatorname{lmo}_{i n v}\left(\mathbb{T}^{2}\right)$ as follows.

Definition 2.8. A function $b \in L^{2}\left(\mathbb{T}^{2}\right)$ is in $\mathrm{lmo}_{\text {inv }}\left(\mathbb{T}^{2}\right)$ if there is a constant $C>0$ such that $\|b(\cdot, t)\|_{*, \log , 1} \leq C$ for all $t \in \mathbb{T}$ and $\|b(s, \cdot)\|_{*, \log , 1} \leq C$ for all $s \in \mathbb{T}$.

Clearly, $l \mathrm{mo}_{i n v}\left(\mathbb{T}^{2}\right)$ is a subspace of $\mathrm{lmo}_{m}\left(\mathbb{T}^{2}\right)$. The one parameter intuition and the equivalent definition of bmo $\left(\mathbb{T}^{2}\right)$ in Proposition 2.1 may lead one to claim that any function $\phi \in L^{\infty}\left(\mathbb{T}^{2}\right) \cap \operatorname{lmo}_{\text {inv }}\left(\mathbb{T}^{2}\right)$ is a multiplier of bmo $\left(\mathbb{T}^{2}\right)$. This is not the case as the above results show and since $L^{\infty}\left(\mathbb{T}^{2}\right) \cap 1 \mathrm{mo}_{\text {inv }}\left(\mathbb{T}^{2}\right)$ contains more than constants. For example, for any $\phi_{1}, \phi_{2} \in L^{\infty}(\mathbb{T}) \cap \operatorname{LMO}(\mathbb{T})$, the function $\phi:(s, t) \mapsto \phi_{1}(s) \phi_{2}(t)$ belongs to $L^{\infty}\left(\mathbb{T}^{2}\right) \cap 1 \operatorname{lmo}_{i n v}\left(\mathbb{T}^{2}\right)$.

2.2. Proof of Theorem 1.5. We prove Theorem 1.5 in this section.

Proof of Theorem 1.5. (i) $\Rightarrow$ (ii): we start by proving that any multiplier from bmo $\left(\mathbb{T}^{N}\right)$ to $\mathrm{bmo}_{m}\left(\mathbb{T}^{N}\right)$ is a bounded function. We recall the following estimate of the mean over a rectangle of functions in $\operatorname{bmo}_{m}\left(\mathbb{T}^{N}\right)$ :

$\left|m_{R} b\right| \lesssim\left(\log \frac{4}{\left|I_{1}\right|}+\cdots+\log \frac{4}{\left|I_{N}\right|}\right)\|b\|_{\text {bmo } m_{m}\left(\mathbb{T}^{N}\right)}, \quad R=I_{1} \times \cdots \times I_{N} \subset \mathbb{T}^{N}$.

It follows that if $\phi$ is multiplier from $\mathrm{bmo}\left(\mathbb{T}^{N}\right)$ to $\mathrm{bmo}_{m}\left(\mathbb{T}^{N}\right)$, then for any $b \in \operatorname{bmo}\left(\mathbb{T}^{N}\right)$ and for any rectangle $R=I_{1} \times \cdots \times I_{N} \subset \mathbb{T}^{N}$,

$$
\begin{aligned}
\left|m_{R}(b \phi)\right| & \lesssim\left(\sum_{j=1}^{N} \log \frac{4}{\left|I_{j}\right|}\right)\|b \phi\|_{\mathrm{bmo}_{m}\left(\mathbb{T}^{N}\right)} \\
& \leq C\left(\sum_{j=1}^{N} \log \frac{4}{\left|I_{j}\right|}\right)\left\|M_{\phi}\right\|_{\mathrm{bmo}\left(\mathbb{T}^{N}\right) \rightarrow \mathrm{bmo}_{m}\left(\mathbb{T}^{N}\right)}\|b\|_{\mathrm{bmo}\left(\mathbb{T}^{N}\right)} .
\end{aligned}
$$

Applying (2.6) to $b=\log _{I_{1}}+\cdots+\log _{I_{N}}$ and using assertions (i) and (ii) of Lemma 2.3, we see that there is a constant $C>0$ such that

$$
\left|m_{R} \phi\right| \leq C\left\|M_{\phi}\right\|_{\mathrm{bmo}\left(\mathbb{T}^{N}\right) \rightarrow \mathrm{bmo}_{m}\left(\mathbb{T}^{N}\right)} \text {, for any } R=I_{1} \times \cdots \times I_{N} \subset \mathbb{T}^{N} .
$$

We conclude that $\phi \in L^{\infty}\left(\mathbb{T}^{N}\right)$.

To prove that $\phi \in \operatorname{lmo}_{m}\left(\mathbb{T}^{N}\right)$, we only need by the definition of $1 \mathrm{mo}_{m}\left(\mathbb{T}^{N}\right)$ to check that for any integer $0<M<N$, any rectangle $R \subset \mathbb{T}^{M}$, $\left\|m_{R} \phi\right\|_{*, \log , K}(K=N-M)$ is uniformly bounded. Let $S$ be a rectangle 
in $\mathbb{T}^{K}$ and $\log _{S}\left(t_{1}, \cdots, t_{K}\right)=\log _{S_{1}}\left(t_{1}\right)+\cdots+\log _{S_{K}}\left(t_{K}\right), S_{j} \subset \mathbb{T}$ be again the associated sum of functions which are uniformly in $\operatorname{BMO}(\mathbb{T})$. We have

$$
\begin{aligned}
L & :=\frac{\sum_{j=1}^{K} \log \frac{4}{\left|S_{j}\right|}}{\left|S_{j}\right|} \int_{S}\left|m_{R} \phi(t)-m_{S \times R} \phi\right| d t \\
& =\frac{1}{|S|} \int_{S}\left|m_{R}\left(\phi \log _{S}\right)(t)-m_{S \times R}\left(\phi \log _{S}\right)\right| d t \\
& \leq\left\|m_{R}\left(\phi \log _{S}\right)\right\|_{\mathrm{bmo}_{m}\left(\mathbb{T}^{K}\right)} \\
& \leq\left\|\phi \log _{S}\right\|_{\mathrm{bmo}_{m}\left(\mathbb{T}^{N}\right)} \\
& \lesssim\left\|\left.M_{\phi}\right|_{\mathrm{bmo}\left(\mathbb{T}^{N}\right) \rightarrow \mathrm{bmo}_{m}\left(\mathbb{T}^{N}\right)}\right\| \log _{S} \|_{\mathrm{bmo}\left(\mathbb{T}^{N}\right)} \\
& =\left\|M_{\phi}\right\|_{\mathrm{bmo}\left(\mathbb{T}^{N}\right) \rightarrow \mathrm{bmo}_{m}\left(\mathbb{T}^{N}\right)}\left\|\log _{S}\right\|_{\mathrm{bmo}\left(\mathbb{T}^{K}\right)} \\
& \lesssim\left\|M_{\phi}\right\|_{\mathrm{bmo}\left(\mathbb{T}^{N}\right) \rightarrow \mathrm{bmo}_{m}\left(\mathbb{T}^{N}\right)} .
\end{aligned}
$$

Hence for any integer $0<M<N$ and for any $R \subset \mathbb{T}^{M},\left\|m_{R} \phi\right\|_{*, \log , N-M}$ is uniformly bounded. Thus, by definition, $\phi \in \operatorname{lmo}_{m}\left(\mathbb{T}^{N}\right)$.

(ii) $\Rightarrow$ (i): Let $\phi \in L^{\infty}\left(\mathbb{T}^{N}\right) \cap \operatorname{lmo}_{m}\left(\mathbb{T}^{N}\right)$. To prove that

$\phi \in \mathcal{M}\left(\operatorname{bmo}\left(\mathbb{T}^{N}\right), \operatorname{bmo}_{m}\left(\mathbb{T}^{N}\right)\right)$, we only need to check that for any integer $0<M<N$, for any rectangle $R \subset \mathbb{T}^{M}$, and any $f \in \operatorname{bmo}\left(\mathbb{T}^{N}\right)$, $\left\|m_{R}(\phi f)\right\|_{*, K}(K=N-M)$ is uniformly bounded. Let $S$ be a rectangle in $\mathbb{T}^{K}$. Then

$$
\begin{aligned}
L & :=\frac{1}{|S|} \int_{S}\left|m_{R}(\phi f)(t)-m_{S \times R} \phi m_{S \times R} f\right| d t \\
& \leq \frac{1}{|S|} \int_{S}\left|m_{R}\left[\left(\phi-m_{S \times R} \phi\right)\left(f-m_{S \times R} f\right)\right](t)\right| d t \\
& +\frac{1}{|S|} \int_{S}\left|\left(m_{S \times R} f\right)\left(m_{R} \phi\right)(t)-m_{S \times R} \phi m_{S \times R} f\right| d t \\
& +\frac{1}{|S|} \int_{S}\left|\left(m_{S \times R} \phi\right)\left(m_{R} f\right)(t)-m_{S \times R} \phi m_{S \times R} f\right| d t \\
& =L_{1}+L_{2}+L_{3} .
\end{aligned}
$$

To estimate the first term, we only use that $\phi \in L^{\infty}\left(\mathbb{T}^{N}\right)$ to obtain

$$
\begin{aligned}
& L_{1}:=\frac{1}{|S|} \int_{S}\left|m_{R}\left[\left(\phi-m_{S \times R} \phi\right)\left(f-m_{S \times R} f\right)\right](t)\right| d t \\
\leq & \frac{1}{|S||R|} \int_{S \times R}\left|\left[\left(\phi-m_{S \times R} \phi\right)\left(f-m_{S \times R} f\right)\right]\left(t_{1}, \cdots, t_{N}\right)\right| d t_{1} \cdots d t_{N} \\
\leq & \frac{\|\phi\|_{L^{\infty}\left(\mathbb{T}^{N}\right)}}{|S||R|} \int_{S \times R}\left|f\left(t_{1}, \cdots, t_{N}\right)-m_{S \times R} f\right| d t_{1} \cdots d t_{N} \\
\leq & \|\phi\|_{L^{\infty}\left(\mathbb{T}^{N}\right)}\|f\|_{\mathrm{bmo}\left(\mathbb{T}^{N}\right)} .
\end{aligned}
$$


For the second term, we use the fact that as $\left\|m_{R} f\right\|_{*, K}$ is uniformly bounded,

$$
\begin{aligned}
\left|m_{S \times R} f\right|=\left|m_{S}\left(m_{R} f\right)\right| & \lesssim\left(\sum_{j=1}^{K} \log \frac{4}{\left|S_{j}\right|}\right)\left\|m_{R} f\right\|_{*, K} \\
& \leq\left(\sum_{j=1}^{K} \log \frac{4}{\left|S_{j}\right|}\right)\|f\|_{\mathrm{bmo}_{m}\left(\mathbb{T}^{N}\right)}
\end{aligned}
$$

$S=S_{1} \times \cdots \times S_{K} \subset \mathbb{T}^{K}, K=N-M$. Consequently,

$$
\begin{aligned}
L_{2} & :=\frac{1}{|S|} \int_{S}\left|\left(m_{S \times R} f\right)\left(m_{R} \phi\right)(t)-m_{S \times R} \phi m_{S \times R} f\right| d t \\
& \lesssim \frac{\left(\sum_{j=1}^{K} \log \frac{4}{\left|S_{j}\right|}\right)\|f\|_{\mathrm{bmo}\left(\mathbb{T}^{N}\right)}}{|S|} \int_{S}\left|\left(m_{R} \phi\right)(t)-m_{S \times R} \phi\right| d t \\
& \leq\|f\|_{\left.\mathrm{bmo} m_{(\mathbb{T}}\right)}\left\|m_{R} \phi\right\|_{*, \log , K} \\
& \leq\|f\|_{\mathrm{bmo}_{m}\left(\mathbb{T}^{N}\right)}\|\phi\|_{{\text {Imom }\left(\mathbb{T}^{N}\right)} .}
\end{aligned}
$$

The last term only uses the fact that $\phi \in L^{\infty}\left(\mathbb{T}^{N}\right)$.

$$
\begin{aligned}
L_{3} & :=\frac{1}{|S|} \int_{S}\left|\left(m_{S \times R} \phi\right)\left(m_{R} f\right)(t)-\left(m_{S \times R} \phi\right)\left(m_{S \times R} f\right)\right| d t \\
& \leq\|\phi\|_{L^{\infty}\left(\mathbb{T}^{N}\right)} \frac{1}{|S|} \int_{S}\left|\left(m_{R} f\right)(t)-m_{S \times R} f\right| d t \\
& \leq\|\phi\|_{L^{\infty}\left(\mathbb{T}^{N}\right)}\left\|m_{R} f\right\|_{*, K} \\
& \leq\|\phi\|_{L^{\infty}\left(\mathbb{T}^{N}\right)}\|f\|_{\mathrm{bmo}_{m}\left(\mathbb{T}^{N}\right)} .
\end{aligned}
$$

The estimates of $L_{1}, L_{2}$ and $L_{3}$, and Lemma 2.2 allow to conclude that

$$
\|\phi f\|_{\mathrm{bmom}_{m}\left(\mathbb{T}^{N}\right)} \lesssim\left(\|\phi\|_{L^{\infty}\left(\mathbb{T}^{N}\right)}+\|\phi\|_{\mathrm{Imom}_{m}\left(\mathbb{T}^{N}\right)}\right)\|f\|_{\mathrm{bmo}\left(\mathbb{T}^{N}\right)} .
$$

This complete the proof of the theorem.

\section{Multipliers to bmo $\left(\mathbb{T}^{N}\right)$}

We would like to deduce some consequences of the above approach. We consider multipliers from any Banach space of functions on $\mathbb{T}^{N}$ (strictly) containing bmo $\left(\mathbb{T}^{N}\right)$ to bmo $\left(\mathbb{T}^{N}\right)$. We have the following general result.

Theorem 3.1. Let $X$ be any Banach space of functions on $\mathbb{T}^{N}$ that strictly contains bmo $\left(\mathbb{T}^{N}\right)$. Then $\mathcal{M}\left(X, \operatorname{bmo}\left(\mathbb{T}^{N}\right)\right)=\{0\}$.

Proof. Clearly, 0 sends any function of $X$ to $\mathrm{bmo}\left(\mathbb{T}^{N}\right)$ by multiplication. Now let $\phi$ be any multiplier from $X$ to bmo $\left(\mathbb{T}^{N}\right)$, then $\phi$ is also a multiplier from bmo $\left(\mathbb{T}^{N}\right)$ to itself. It follows from Theorem 1.3 that $\phi$ is a constant $C$. Suppose that $C \neq 0$ and recall that $\mathrm{bmo}\left(\mathbb{T}^{N}\right)$ is a proper subspace of $X$. Then for any $f \in X$, we have that $f=C\left(\frac{1}{C} f\right)=\phi\left(\frac{1}{C} f\right) \in \operatorname{bmo}\left(\mathbb{T}^{N}\right)$. 
This contradicts the fact that $\operatorname{bmo}\left(\mathbb{T}^{N}\right)$ is a strict subspace of $X$. Hence $C$ is necessarily 0 . The proof is complete.

Taking as $X$, the Chang-Fefferman BMO space or $\operatorname{bmo}_{m}\left(\mathbb{T}^{N}\right)$ we have as corollary the following.

Corollary 3.2. We have

$$
\mathcal{M}\left(\operatorname{BMO}\left(\mathbb{T}^{N}\right), \operatorname{bmo}\left(\mathbb{T}^{N}\right)\right)=\mathcal{M}\left(\operatorname{bmo}_{m}\left(\mathbb{T}^{N}\right), \operatorname{bmo}\left(\mathbb{T}^{N}\right)\right)=\{0\} .
$$

The author would like to thank the referee for comments and observations that improved the presentation of this note.

\section{REFERENCES}

[1] S-Y. A. Chang and R. Fefferman, A continuous version of $H^{1}$ duality with BMO on the bidisc, Ann. of Math. (2) 112 (1980), no. 1, 179-201.

[2] M. Cotlar, C. Sadosky, Two distinguished subspaces of product BMO and Nehari-AAK theory for Hankel operators on the torus, Integral Equations Operator Theory 26 (1996), no. 3, 273304.

[3] S. Ferguson, C. Sadosky, Characterizations of bounded mean oscillation on the polydisk in terms of Hankel operators and Carleson measures, J. Anal. Math. 81 (2000), 239-267.

[4] S. Pott, B. Sehba, Logarithmic mean oscillation on the polydisc, endpoint results for multi-parameter paraproducts, and commutators on BMO, J. Anal. Math. 117 (2012), No. 1, 1-27.

[5] S. Pott, B. Sehba, The multiplier algebra of product BMO, preprint.

[6] B. F. Sehba, Operators on some analytic function spaces and their dyadic counterparts, thesis, University of Glasgow 2009.

[7] D. A. Stegenga, Bounded Toeplitz operators on $H^{1}$ and applications of the duality between $H^{1}$ and the functions of bounded mean oscillation, Amer. J. Math. 98 (1976), No. 3, 573-589

[8] B. Wick, Little BMO and Muckenhoupt weights in product domains, unpublished notes.

E-mail address: bsehba@gmail.com 